\title{
AC 2010-2017: THREE PRACTICAL DEMONSTRATIONS IN BEEM PROJECT
}

Huihui Xu, Rose-Hulman Institute of Technology

Xiaoyan Mu, Southeast Missouri State University

Deborah Walter, Rose-Hulman Institute of Technology 


\title{
Three Practical Demonstrations in BEEM Project
}

\begin{abstract}
This paper presents three practical examples that have been created in the $\mathrm{BEEM}^{1}$ (Biomedical and Electrical Engineering Methods) project at Rose-Hulman Institute of Technology. These examples are used to introduce respectively (1) Construction of a prototype electrocardiogram measurement system, (2) Use of inductance coils to perform as transformers, and (3) Audio signal filter. These demonstrations are easy and inexpensive to implement and perform. They have been used to help students understand the underlying electrical principles in addition to demonstrating measurement techniques and solution options for the topics discussed in related electrical and/or biomedical engineering courses. By the means of demonstrating the real-world applications, they should prove useful in improving students' learning and interest in electrical engineering principles and applications.
\end{abstract}

\section{Introduction}

Electrical engineering topics are perceived by many students as theoretical and not related to real world applications. In particular, this abstract nature has been identified as one reason for the small number of women that are attracted to electrical engineering major. Female undergraduates prefer human-centered majors and applications that help people ${ }^{2}$. On the other hand, electrical engineering fundamentals are required for the biomedical engineering major, particularly for the advancement of biomedical sensors and instrumentation. Applications incorporating the life science and biological principles into practical medical devices and systems are desired by biomedical engineering students to spur their interest in electrical engineering topics.

To stimulate the students' interest and change the fact that the electrical engineering coursework is underappreciated, the instructor often present real world applications in the form of demonstrations in class. The development and set-up of these demonstrations can be highly time-consuming. The purpose of this paper is to enhance the experimental materials for demonstrating the real-world applications of electrical engineering principles. All demonstrations can be performed easily and inexpensively. For each demonstration, the background on the real-world application, the learning objectives, the design process and system component, and the set-up and testing of the final system are explained. These experiments work together to expose the students to several common topics of fundamental electrical engineering classes. The experiments have been used either as in-class demonstrations or as student laboratory exercises in required sophomore-level courses. They have helped to enhance students' understanding of the underlying electrical principles, problem characteristics, solution options, and measurement techniques for the topics under discussion. The physical demonstrations of the presented examples are video-taped, and are available on the project BEEM website ${ }^{3}$. 


\section{Demonstration \#1: Construction of a Prototype ECG Measurement System}

A home-made prototype electrocardiogram (ECG) measurement system is the most essential component of this demonstration. The system includes an ECG surface electrode, a signal conditioner, an analog-to-digital (A/D) converter, and MATLABß-based signal processing module on a computer. This demonstration enhances the students' understanding of how the fundamental electrical engineering concepts can be integrated with the principles of life science to implement a practical medical device that benefits our life.

\subsection{Learning objectives}

The ECG demonstration introduces the basic process of a rather complicated biomedical-related measurement system. The bio-electrical signal originates from a human subject and is extracted using standard $\mathrm{Ag} / \mathrm{AgCl}$ surface electrodes. Recording the $\mathrm{ECG}$, and biopotentials in general, creates special requirements for the signal conditioning circuit because the skin potentials related to heart tissue activity, which make up the ECG, are small (a few millivolts or less). Therefore an amplifier with high gain, usually $500-1000$, is needed. The amplifier must also have high input impedance in order to avoid loading the signal source. The frequency component of the bioelectrical signal is another important characteristic. The frequency range of the ECG is well established $(0.01-250 \mathrm{~Hz})$, which allows us to design filters in the signal conditioner to attenuate signal components outside this bandwidth. This demonstration also involves the use of an analog-to-digital conversion module for displaying and processing the digital ECG wave signals on a computer.

After practicing with this demonstration, students should be able to:

- Understand the electrical characteristics of common biological signals;

- Describe the design specification of the signal conditioner circuit;

- Recognize major ECG waveforms;

- Understand and implement the analog-to-digital conversion.

\subsection{Equipment}

The following table lists the instruments needed to perform this demonstration. Most of the equipment is commonly found in an electronics laboratory. The only appliances that may not be common are the Measurement Computing data acquisition module and the ECG surface electrodes. Note that the Analog Device's AD620 is a monolithic version of the three op-amp differential amplifier and the Burdick ${ }^{\circledR} \mathrm{EKG}$ is optional.

\begin{tabular}{|l|}
\hline \multicolumn{1}{|c|}{ Table 1: Instruments used in the ECG demonstration } \\
\hline Agilent dual DC power supply \\
\hline Agilent 0-20 MHz function generator \\
\hline Agilent 0-100 MHz oscilloscope \\
\hline AD620, 741/347 op-amps \\
\hline Breadboard, BNC banana connectors, and testing cables \\
\hline Resistor and capacitor assortment \\
\hline Measurement Computing USB 1608FS data acquisition module \\
\hline ECG surface electrodes, Burdick $®$ EK-10 EKG (optional) \\
\hline
\end{tabular}




\subsection{Construction and testing of the biopotential amplifier}

Figure 1 shows a sample schematic of the ECG amplifier. An instrumentation amplifier with a differential-mode gain of 25 is designed and constructed using Analog Device's AD620. The AD620 is used because it offers high input impedance and high common mode rejection ratio, and being monolithic the AD620 specifications are far superior to those of any discrete version. Two $\mathrm{Ag} / \mathrm{AgCl}$ surface electrodes are placed on the left and right arm of a volunteer to acquire the skin biopotential of a lead-I ECG, i.e., the differential signal from left arm to right arm.

The instrumentation amplifier is followed by an active band-pass filter. The band-pass filter attenuates the signals that are out of the frequency range according to the ECG frequency specifications. An inverting amplifier is used to compensate the negative gain introduced by the band-pass filtering and produce additional gain that is necessary before signal digitization.

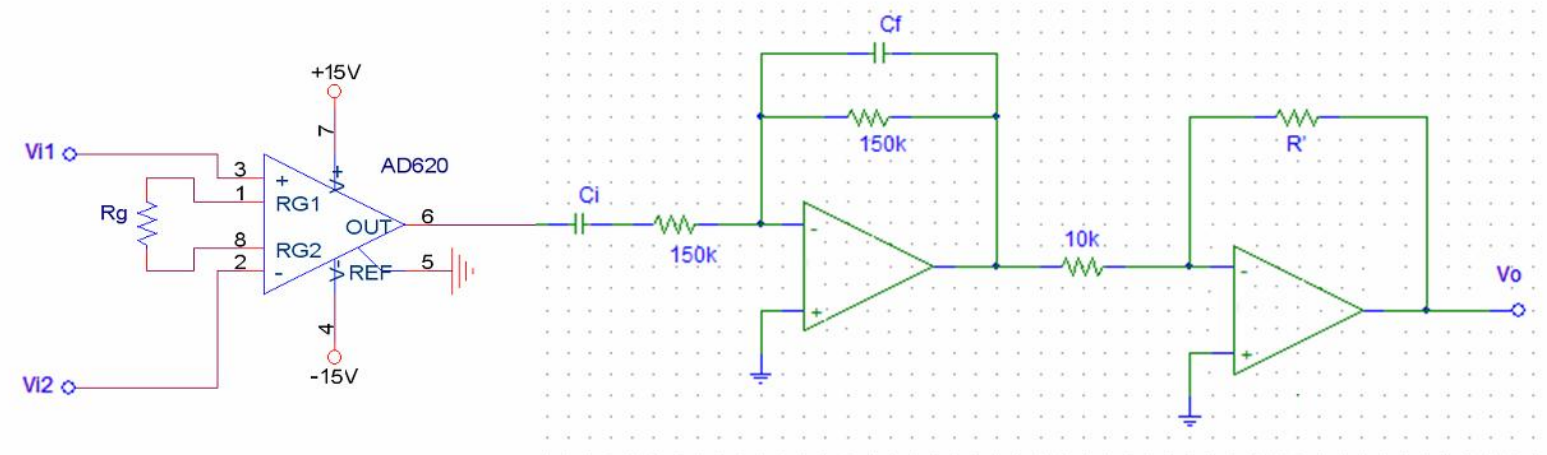

Figure 1: A sample schematic of an ECG amplifier

For testing the biopotential conditioning circuit, several factors are evaluated: the differentialmode gain and the common mode rejection ratio of the instrumentation amplifier, the cutoff frequencies and the frequency response of the band-pass filter, the amplification factor of the inverting amplifier. The amplification and filtering performances of the complete biopotential amplifier circuit are also evaluated as one system.

\subsection{Testing of the prototype measurement system}

In this demonstration, $\mathrm{A} / \mathrm{D}$ conversion is attained using a USB-based data acquisition (DAQ) module made by Measurement Computing (model USB 1608FS), Figure 2 (a). The ECG signal is extracted by $\mathrm{Ag} / \mathrm{AgCl}$ surface electrodes placed on two arms of a human subject. Skin electrical signals are acquired by the two input pins of the ECG biopotential amplifier (i.e., the two inputs of the instrumentation amplifier), through which the signals are amplified and filtered. The A/D converter digitizes the signals and finally the digital ECG signals are displayed on the screen. Communication between the biopotential circuit and DAQ device is through a MATLAB ${ }^{\circledR}$ program, which is coded to acquire the signal data for several seconds at a sampling rate of a few thousand Hertz. 
A simple two-lead plus ground configuration (an electrode placed on the right leg is used as a ground) may be implemented in the demonstration to measure lead-I ECG, as shown in Figure 2 (b). The circuit can be easily modified if other frontal leads, e.g., lead-II, lead-III, of ECG are desired. It is also possible to use a BNC T-connector to display the output signal on an oscilloscope as well as the computer. A photograph of the circuit set-up is shown in Figure 2 (c).

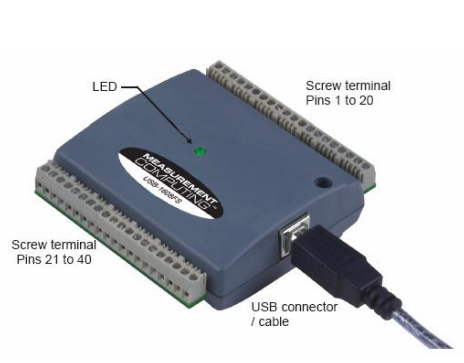

(a)

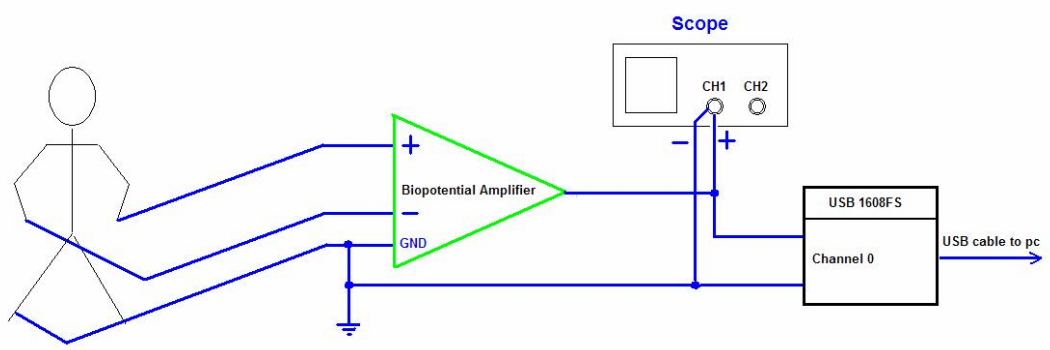

(b)

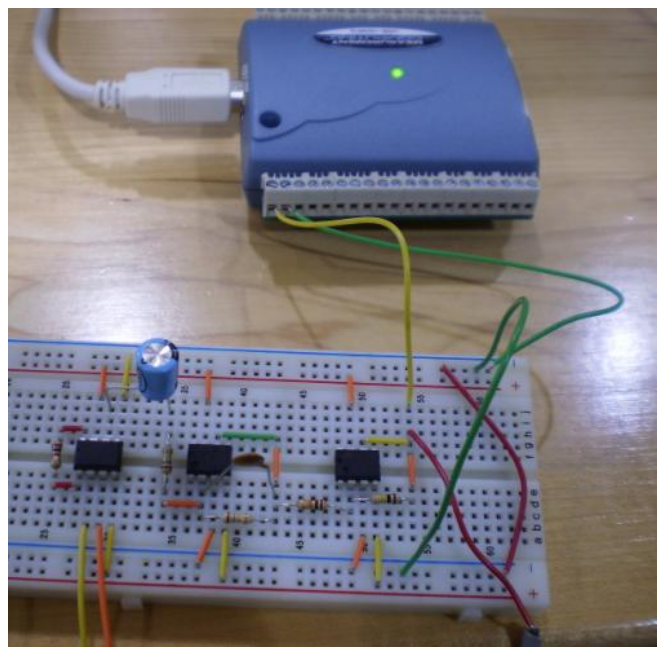

(c)

Figure 2: (a) Measurement Computing USB A/D converter; (b) ECG acquisition system set-up; and (c) a photograph of the ECG biopotential circuit with connections to the A/D converter.

With successful implementation of a prototype ECG measurement system, the P, QRS complex, and $\mathrm{T}$ waveforms may be clearly recognized in a digital format. Suggested post-demo exercises include estimating the heart rate and digital signal processing such as filtering. A portable Burdick ${ }^{\circledR}$ EKG may be used to compare to the results of the prototype ECG system.

\section{Demonstration \#2: Use of Inductance Coils to Perform as Transformers}

It has been observed in the electrical circuits' class that students often have difficulties understanding the concept of mutual inductance because it seems abstract to them. The purpose of this demonstration is to help students develop intuition regarding the concept of mutual inductance and understanding of one common application: transformers. 


\subsection{Learning objectives}

This demonstration has two major components which aim to help students learn two important topics of electrical circuits: mutual inductance and transformer. The first part of the demonstration involves a mutual inductance circuit which consists of two loops. The second part of the demonstration showed the students what is inside a real transformer and how it is linked to the concept of mutual inductance.

After observing the classroom demonstration, students should be able to:

- Understand mutual inductance;

- Understand how the mutual inductance concept is linked to its application - transformer.

\subsection{Equipment}

Table 2 lists the parts and equipment needed to perform this demonstration.

\begin{tabular}{|l|}
\hline \multicolumn{1}{|c|}{ Table 2: Instruments used in the Transformer Demonstration } \\
\hline Agilent $0-220 \mathrm{~V} \mathrm{AC}$ power supply \\
\hline Agilent $0-20 \mathrm{MHz}$ function generator \\
\hline Agilent $0-100 \mathrm{MHz}$ oscilloscope \\
\hline Two electrical coils \\
\hline Light bulb \\
\hline CHNT NDK(BK)-50 50W 110-220V Transformer \\
\hline BNC banana connectors, and testing cables \\
\hline
\end{tabular}

\subsection{Construction of the demonstration}

The demonstration is divided into two parts. This fist part is to demonstrate a mutual inductance circuit, which involves two loops. The first loop is composed of a time-varying voltage source and a coil while the second loop has a coil and a lamp. Figure 3 (a) shows the construction of the mutual inductance circuit. When the two coils are place close to each other, the mutually induced voltage energizes the lamp connected to the second coil, as shown in Figure 3 (b).

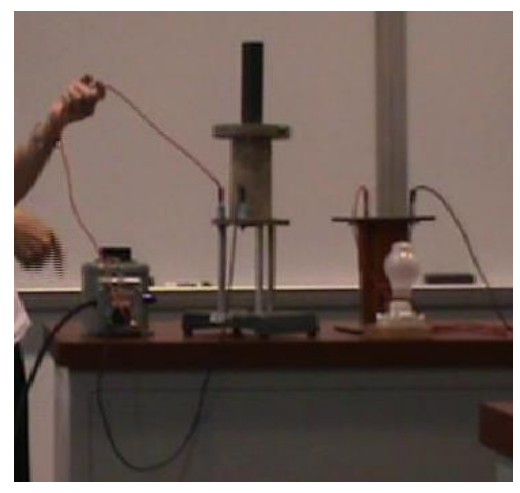

(a)

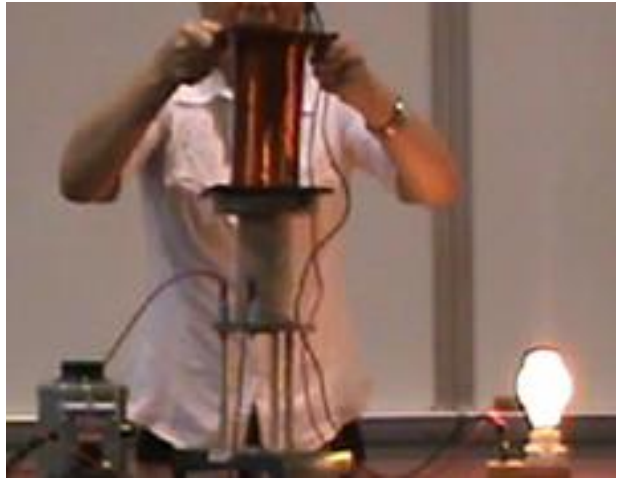

(b)

Figure 3: (a) Construction of the mutual inductance circuit; (b) Mutually induced voltage energizes up the lamp connected to the secondary coil in the mutual inductance circuit. 
The second part of the demo shows that a transformer is a pair of mutually coupled coils and the turn's ratio of the coils is related to the ratio of the primary and secondary voltages. A laminated core transformer is deconstructed intentionally so that the students can observe the primary and secondary coils inside the transformer. The primary coil is energized by a common 110Vac power supply. The voltages of the primary and secondary coils are displayed on the oscilloscope and RMS values are measured.

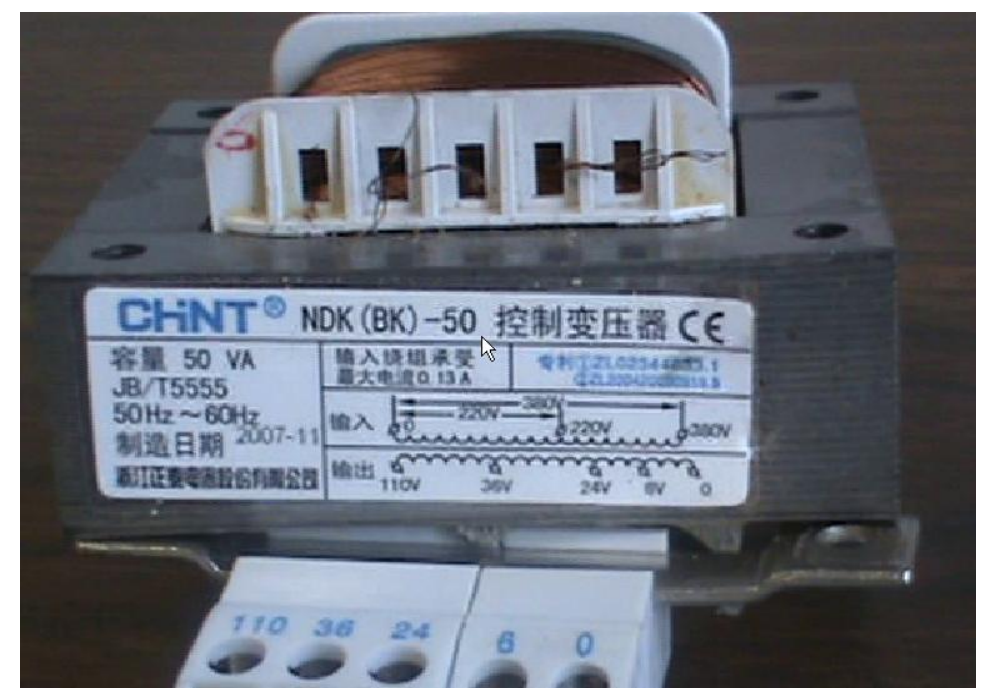

Figure 4: CHNT NDK(BK)-50 50W 110-220V transformer

\section{Demonstration \#3: Audio Signal Filter}

This demonstration is used to elucidate the application of filters to a broadband signal. Audio signals are used to develop an intuitive understanding of the concept of filtering. Referring to the schematic in Figure 5, an audio signal is converted to an electrical signal. The electrical signal (original signal) is input to a filter (a low-pass, high-pass, band-pass, or band-reject filter). The output of the filter (filtered signal) is converted back to an audio signal and the students are able to hear the difference in the spectral quality of the signals by playing both the input and output audio signals over a speaker.

\subsection{Learning objectives}

Much of the study of electrical signals and systems rely on a conceptual understanding of the characteristics of the time-dependent, and frequency-dependent nature of signals and the relationships between frequency content and transient characteristics. Electrical signals are not visible, but students generally have a lot of experience with waves, so the understanding of the time varying sinusoidal signal is a relatively easy concept to grasp. However, frequency characteristics are often more difficult for students to understand because they do not have a lot of experience with visualizing the frequency of a signal. A typical example used to help students visualize the effects of variable frequency of a signal is to use a sound wave with a changing pitch. This demonstration and the associated classroom activities are developed to demonstrate the relationship between time and frequency domain representations of signals. 
After observing the classroom demonstrations, students should be able to:

- Identify the frequency characteristics of a broadband signal displayed in the time-domain;

- Describe the similarities of frequency changes in an electrical signal and frequency changes in an audio signal;

- Predict the qualitative changes to a broadband signal after it has been passed through a low-pass, high-pass, band-pass, or band-reject filter;

- State practical applications for an electrical signal filter.

\subsection{Equipment}

Most of the equipment used for this demonstration is commonly found in an electronics laboratory. Table 3 lists the parts and equipment needed to perform this demonstration.

\begin{tabular}{|l|}
\hline Table 3: Instruments used in the Audio Signal Filter Demonstration \\
\hline Agilent dual DC power supply \\
\hline Agilent $0-100 \mathrm{MHz}$ oscilloscope \\
\hline TL072 op-amps \\
\hline Breadboard, BNC banana connectors, and testing cables \\
\hline Resistor and capacitor assortment \\
\hline Two speakers \\
\hline MP3/4 player \\
\hline
\end{tabular}

\subsection{Construction and demonstration set-up}

One main component of the system is an input signal supplied by an MP3/4 player or a computer. The input signal is followed by a series of filters. Both the original signal and the filtered signal are output to a speaker and displayed on an oscilloscope, where the oscilloscope displays the time-domain characteristics of the signals that can be seen by the eye and the speakers are used to demonstrate the frequencies of the signals that can also be heard with the ear. The filter system includes a low-pass, a high-pass, a band-pass, and a band-reject filter. The low- and high-pass filters are cascaded to create a broadband band-pass filter. The band-reject filter is twin-T notch filter with a variable resistor to control the bandwidth. The bandwidth is approximately $32(1-\sigma) \mathrm{kHz}$. Table 4 summarizes the characteristics of the four different filters, where $\alpha$ is variable, between 0 and 1 , that is controlled with a potentiometer. A picture of the measurement set-up in the classroom is shown in Figure 6.

\begin{tabular}{|l|l|l|}
\hline \multicolumn{3}{|c|}{ Table 4: Filter Characteristics } \\
\hline Filter & Frequency selection & Pass-band gain \\
\hline Low-pass & Cutoff frequency $=160 \mathrm{kHz}$ & 100 \\
\hline High-pass & Cutoff frequency $=340 \mathrm{~Hz}$ & 100 \\
\hline \multirow{2}{*}{ Band-pass } & Center frequency $=7.3 \mathrm{kHz}$ & \multirow{2}{*}{100,000} \\
\cline { 2 - 3 } & Corner frequencies $=340 \mathrm{~Hz}, 160 \mathrm{kHz}$ & \multirow{2}{*}{1} \\
\hline \multirow{2}{*}{ Band-reject } & Center frequency $=7.9 \mathrm{kHz}$ & \multirow{2}{*}{ Bandwidth varies by adjusting $\alpha$} \\
\cline { 2 - 3 } & Band
\end{tabular}




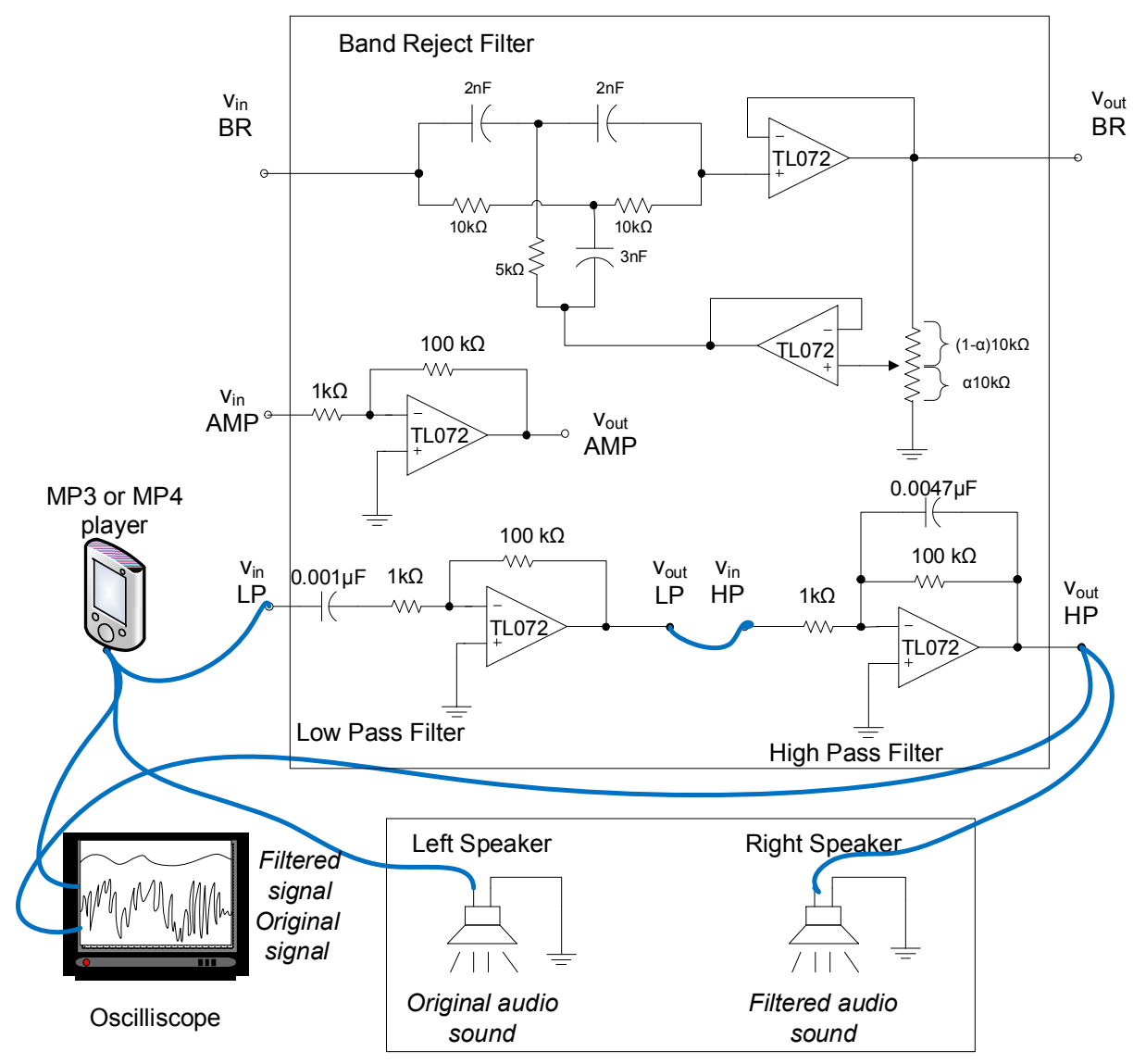

Figure 5: Audio signal filter schematic. Connections are made to send the original and filtered signals to a speaker and to an oscilloscope. The low-pass and high-pass filters are cascaded to create a broadband band-pass filter. Computer speakers with individual volume controls are recommended because they can amplify the signal loud enough for a large room.

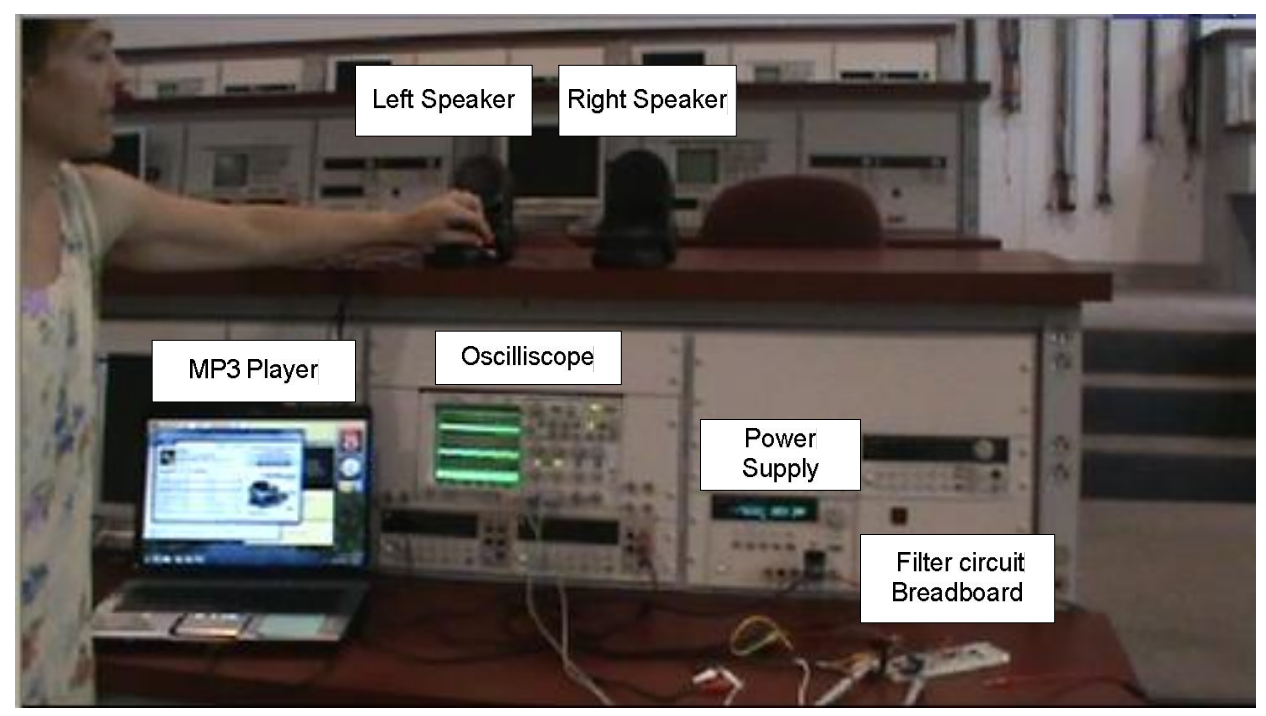

Figure 6: Audio signal filter classroom demonstration set-up 


\subsection{Classroom activities using the audio signal filter}

The classroom activities suggested here are useful for the students to determine the relationship between the time-domain and frequency-domain representations of signals. Figure 7 shows a sample oscilloscope display during the presentation of classroom exercises.

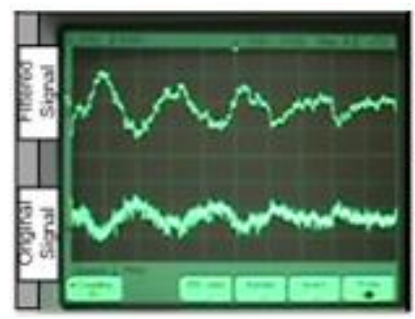

(a) Low-pass filter

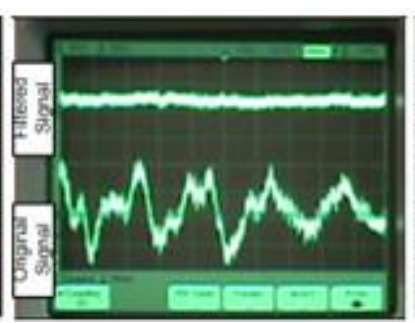

(b) High-pass filter

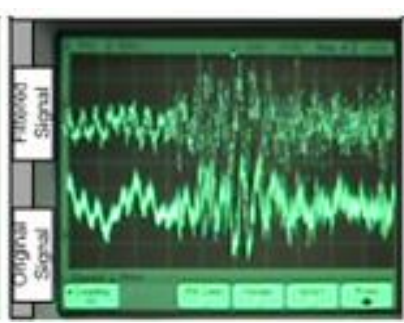

(c) Band-pass filter

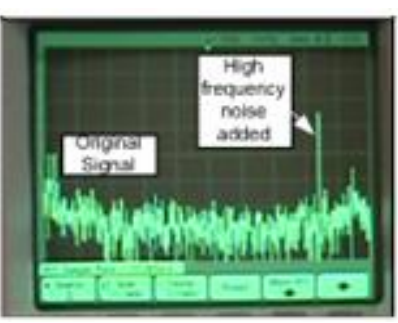

(d) Frequency response

Figure 7: Oscilloscope display of original time-domain signals and the resultant signals after it has been (a) low-pass, (b) high-pass, and (c) band-pass filtered. (d) shows the frequency response of the signal with a very narrow-band high frequency noise signal added.

\subsubsection{Low-pass filter}

Referring to Figure 5, the original signal, i.e., the MP3/4 player's output, is connected to the scope, the left speaker, and the input of the low-pass filter $\left(V_{\text {in }} L P\right)$. The output of the low-pass filter ( $\mathrm{V}_{\text {out }} \mathrm{LP}$ ) is connected to the right speaker and the oscilloscope. The oscilloscope is adjusted to display both the original and filtered signals simultaneously, as shown in Figure 7 (a). Note that the audio signal that the students hear corresponds to the time domain characteristics on the scope. The volume of the sound is related to the amplitude of the signal, low frequencies (like rhythmic drum beats) correspond to larger periodic wavelengths, and high frequencies (like female voices) correspond to short periodic and non periodic signatures. The volume of the original signal and the filtered signal may be adjusted. By having two speakers with individual volume controls, it is easy to switch the audio signal between the filtered and the original signals, so that the students can tune into the characteristic differences in the sound and connect that knowledge to the time-domain differences displayed on the oscilloscope.

\subsubsection{High-pass filter}

MP3/4 player's output is connected to the scope, the left speaker, and the input of the high-pass filter $\left(V_{\text {in }} H P\right)$. The output of the high-pass filter $\left(V_{\text {out }} H P\right)$ is connected to the right speaker and the oscilloscope. One can switch the speakers from the original signal to the filtered signal several times and point out on the oscilloscope display (Figure 7 (b)) that the low frequencies (longer wavelengths) have been filtered out, while the higher frequencies are allowed to pass.

\subsubsection{Band-pass filter}

Since the low- and high-pass filters can be cascaded together to create a broadband band-pass filter, it is often useful to demonstrate the band-pass filter after first showing the effects of the 
low- and high-pass filters independently. Because a higher pass-band gain is selected than that of the low- or high-pass filter, reducing the volume on the speakers may be necessary. This circuit design is a very broad-band filter; most of the original signal's characteristics remain the same. A sample of the time-domain signals is shown in Figure 7 (c).

\subsubsection{Band-reject filter}

For the purpose of demonstrating the effect of the band-reject filter, in this example exercise, a narrow-band noise signal of approximately $8 \mathrm{kHz}$ is synthetically added to the audio file using a MATLAB ${ }^{\circledR}$ program (Figure 7 (d)). The MP3/4 player's output is connected to the scope, the left speaker, and the input of the band-reject filter $\left(\mathrm{V}_{\text {in }} \mathrm{BR}\right)$. Since the pass-band gain of the band-reject filter is unity, also connect in cascade the amplifier circuit ( $V_{\text {out }} B R$ to $\left.V_{\text {in }} A M P\right)$. The output of the amplifier circuit ( $\left.\mathrm{V}_{\text {out }} \mathrm{AMP}\right)$ is connected to the right speaker and the oscilloscope. When the original signal is played over the left speaker, the added noise sounds like a high pitched whine, which can be clearly detected. The oscilloscope is used to display the frequency spectrum of the signal, in which the added noise is clearly distinguished. Then the filtered signal is played. The bandwidth of the filter can be adjusted by the variable resistor. Because a high-Q filter is used, if the filter's notch is not perfectly matched to the added noise (this can easily be due to differences in the parts from their nominal values), the noise can still be distinguished in the filtered signal. As the bandwidth is increased the noise signal gradually fades, with not much distortion in the audio signal.

\section{Conclusion}

Three real-world application demonstrations are presented in this paper. The learning objectives, the design specifications and system component, and the set-up and testing of the final system are included and discussed. The demonstrations are easy and inexpensive to implement and perform.

These demonstrations work together to expose the students to many common topics in electrical engineering such as inverting amplifier, instrumentation amplifier, analog filters (low-pass, highpass, band-pass, and band-reject), analog-to-digital conversion, (mutual) inductance, and transformer. All these demonstrations have been performed as either part of the laboratory portion or in-class demonstrations. We have found they are valuable and effective additions which have helped to enhance student interest and to better understand the underlying electrical concepts in addition to demonstrating the measurement techniques. In the future, student feedback will be collected and analyzed statistically to study the effectiveness of these demonstrations.

\section{Bibliography}

1. Xu, et. al., (2009). BEEM: A project to enhance lab and design skills for BME students. 2009 BMES Annual Meeting

2. Rosser, S. V. (1997). Re-engineering female friendly science. Athene series. New York: Teachers College Press.

3. http://ece-2.rose-hulman.edu/beem/ 